\title{
Los últimos serán los primeros: la importancia de los hablantes de herencia
}

\author{
María Elena Ibáñez Bravo
}

\begin{abstract}
Resumen
La diversidad lingüística se encuentra en grave riesgo ante el desplazamiento de 96\% de lenguas que se hablan a nivel mundial a favor de sólo 3\% de lenguas dominantes. Ante esta pérdida, las acciones de revitalización, fortalecimiento y promoción de lenguas se vuelven fundamentales para intentar revertir dicha situación. En este escenario, los hablantes de herencia, un tipo de bilingüismo recientemente reconocido, se consideran figuras fundamentales para ampliar el panorama de estrategias de planificación lingüística, ya que por las características que presentan es posible aumentar las opciones de espacios de uso y de enseñanza de lenguas a favor de las comunidades de habla que se encuentran en contextos de desplazamiento lingüístico.
\end{abstract}

Palabras clave: hablantes de herencia, derechos lingüísticos, diversidad lingüística, bilingüismo.

\section{THE LAST SHALL BE FIRST: THE IMPORTANCE OF HERITAGE SPEAKERS}

\begin{abstract}
Linguistic diversity is at serious risk due to the displacement of $96 \%$ of globally spoken languages in favor of only $3 \%$ of dominant languages. Faced with such loss, actions to revitalize, strengthen and promote languages become fundamental in order to reverse this situation. In this scenario, heritage speakers, a recently recognized type of bilingualism, are considered essential to broaden the panorama of strategies to be implemented: due to their characteristics it is possible to increase the options of spaces of use and teaching of languages in favor of the ones that are at risk.
\end{abstract}

Keywords: heritage speakers, linguistic rights, linguistic diversity, bilingualism.

Recepción: 03/02/2021. Aprobación: 21/04/2021.

http://doi.org/10.22201/cuaieed.16076079e.2021.22.4.6 
Cursó la licenciatura de lingüística en la Escuela Nacional de Antropología e Historia (ENAH) y la maestría en Lenguas Modernas en la Universidad Autónoma de Baja California. Se ha especializado en el estudio de lenguas yumanas, y en peligro de extinción, especialmente en lenguas paipai, kiliwa y ko'áhl.

Ha laborado, en materia de políticas lingüísticas, en diversas instituciones nacionales como el Instituto Nacional de Lenguas Indígenas (INALI) y el Instituto Nacional de Educación para Adultos (INEA); como tallerista en la Secretaría de Cultura, y ha colaborado con la Biblioteca Vasconcelos para la divulgación de la diversidad lingüística.

También ha impartido clases en la licenciatura de lingüística y de etnología, de la Escuela Nacional de Antropología e Historia (ENAH) y en Gestión cultural en la Universidad Autónoma Benito Juárez de Oaxaca (UABJO).

\section{Rosa y Fernando}

Rosa tenía 3 años cuando toda su familia se fue a vivir a Chicago. Rosa es la menor de cuatro hermanos. En cuanto llegaron a Chicago, entraron a la escuela y ahí empezaron a aprender inglés. Toda su familia aprendió la lengua, no obstante, en casa únicamente se hablaba español. Rosa, a diferencia de sus hermanos, entiende todo el español que se usa en su hogar, pero ella sólo habla en inglés. Cuando intenta hablar español, lo habla muy diferente a su familia y se desespera porque no lo puede hablar igual que ellos. Se siente más cómoda hablando inglés.

Fernando es el mayor de tres hermanos. Ellos se criaron desde chiquitos con su tía - que únicamente hablaba en paipai-, porque su mamá trabajaba afuera de la comunidad. A Fernando le cuentan que de chiquito hablaba paipai, pero que cuando empezó a ir a la escuela toda su familia le empezó a hablar en puro español. Fernando entiende las dos lenguas, el paipai y el español, aunque sólo habla en español. Su familia no quería que lo discriminaran como a ellos y por eso le empezaron a hablar sólo en español, para que pudiera tener más oportunidades de trabajo afuera de la comunidad. Actualmente solamente quedan 35 hablantes de paipai... 


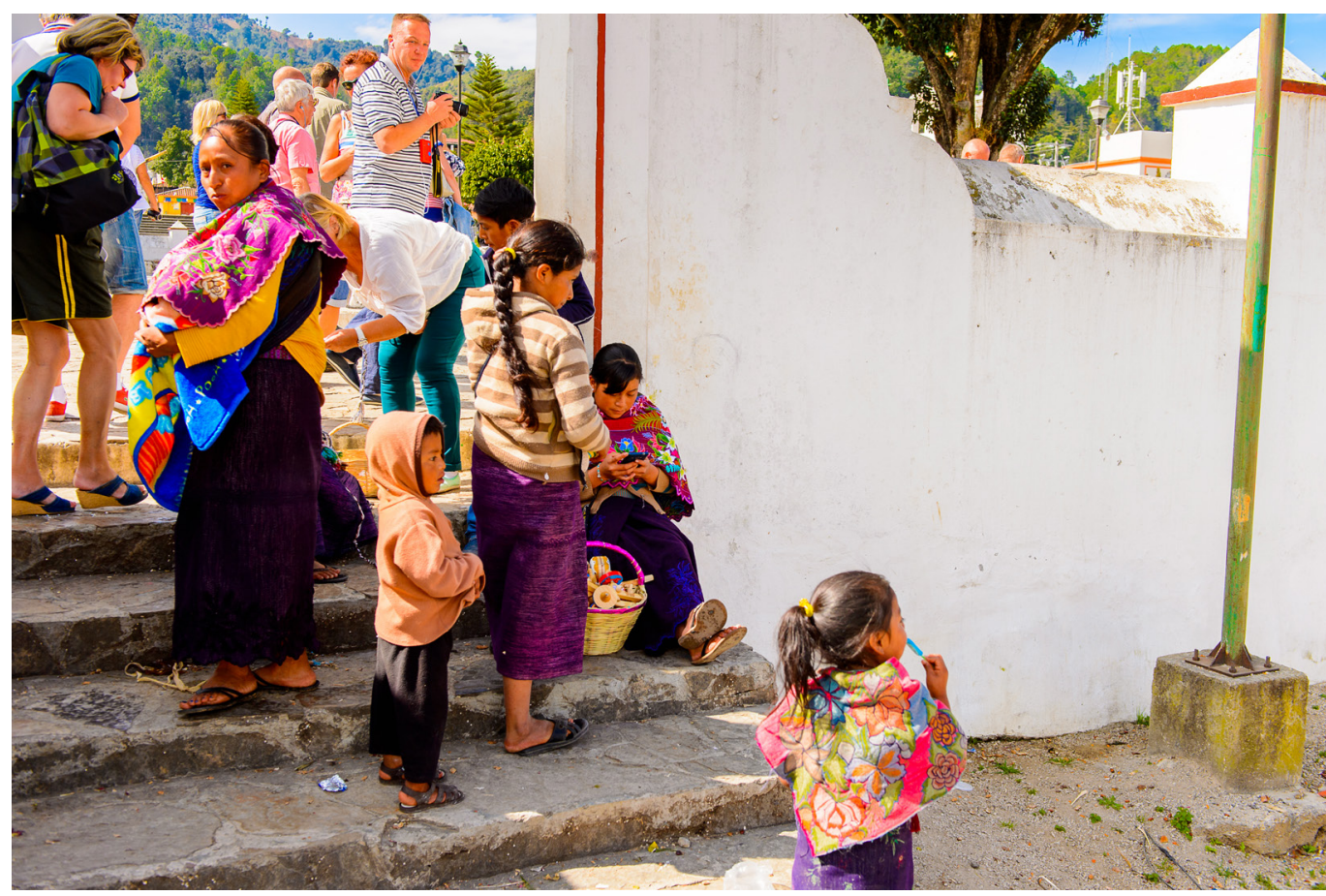

\section{Hablantes de herencia}

En contextos en los que diversas culturas y lenguas conviven, por ejemplo, los de migración o regiones con alta diversidad cultural y lingüística, existen generaciones que sólo hablan en la lengua de más prestigio o dominante, pero entienden dos, la de casa y la dominante. En el caso de Rosa, la lengua de casa es el español y la lengua dominante el inglés, situación que es común en las familias hispanohablantes que viven en Estados Unidos. En cuanto a Fernando, su legua de casa es el paipai, lengua indígena de Baja California, y la dominante es el español.

Al bilingüismo que presentan Rosa y Fernando se le conoce como bilingüismo de hablantes de herencia $(\mathrm{HH})$, y sus contextos de subordinación lingüística corresponden a los casos que se dan en México con la población migrante y con la población indígena originaria. Como menciona Montrul (2016), especialista en el estudio de este tipo de bilingüismo, los HH se pueden dar en otros contextos como el caso de los hijos bilingües de familias en Canadá y en Europa, en donde los niños crecen con la estrategia de un pariente-una lengua, es decir, que el padre y la madre les hablan en lenguas diferentes a sus hijos. También incluyen los casos de adopción internacional, en la que los niños mantienen algún conocimiento de su primera lengua.

Al hablar de bilingüismo es necesario tener claro qué se entiende al usar este término. El bilingüismo se define como un comportamiento lingüístico y social de alternancia de lenguas que usa un hablante de acuerdo a su nivel de domino y a sus necesidades de uso en cada una de las lenguas (Mackey, 1962; Valiñas, 1986). Al ser el bilingüismo un comportamiento en el que influyen diversos factores, para su estudio se identifican diversos tipos, entre los cuales se encuentran los hablantes de herencia. 
El presente ensayo tiene como propósito mostrar un panorama general sobre el bilingüismo de los hablantes de herencia, el cual ha sido recientemente reconocido y estudiado. Asimismo, se busca reflexionar en la enseñanza de las lenguas de herencia como parte de los procesos de fortalecimiento, desarrollo y revitalización de las comunidades de habla que se encuentran en desplazamiento lingüístico, es decir, en contextos de subordinación lingüística por parte de otra comunidad de habla cuya lengua es valorada como la lengua de prestigio o de dominación.

\section{Características de los hablantes de herencia}

A nivel mundial la diversidad lingüística se encuentra en grave riesgo de pérdida. La unEsco (2003) establece que 96\% de las aproximadamente 7000 lenguas que existen a nivel mundial son habladas tan sólo por 3\% de la población mundial. Dicha diversidad se encuentra en grave riesgo debido al desplazamiento lingüístico, que se puede definir como un proceso lento y silencioso en el que los hablantes van modificando actitudes a favor a la lengua dominante (Valiñas, 1986), en el caso de Rosa hacia el inglés y en Fernando hacia el español. Deesta manera, cadavezqueun hablantebilingüe participa en una conversación en la que decide usar la lengua dominante, desplaza gradualmente las reglas y valores que subyacen a su lengua de casa que está siendo subordinada, generando así un cambio en el comportamiento lingüístico de los participantes en la conversación. Al cambiar dichas reglas y valores se modifican las condiciones que sustentan la cultura e ideología histórica de la sociedad dominada. Por lo tanto, hablar de desplazamiento lingüístico, como menciona Valiñas (1986), implica la existencia de una relación de dominio y opresión, que amenaza la reproducción social y cultural de un pueblo, ejerciendo presión constante para su asimilación a la población dominante. El bilingüismo que presentan Rosa y Fernando es el último bilingüismo previo a las generaciones de hablantes que sólo hablan y entienden una lengua, la lengua dominante, con lo que el desplazamiento lingüístico se concreta en un monolingüismo, en la lengua dominante.

Video 1. ¿Qué son los hablantes de herencia? (Cursos Español Colorado, 2019).

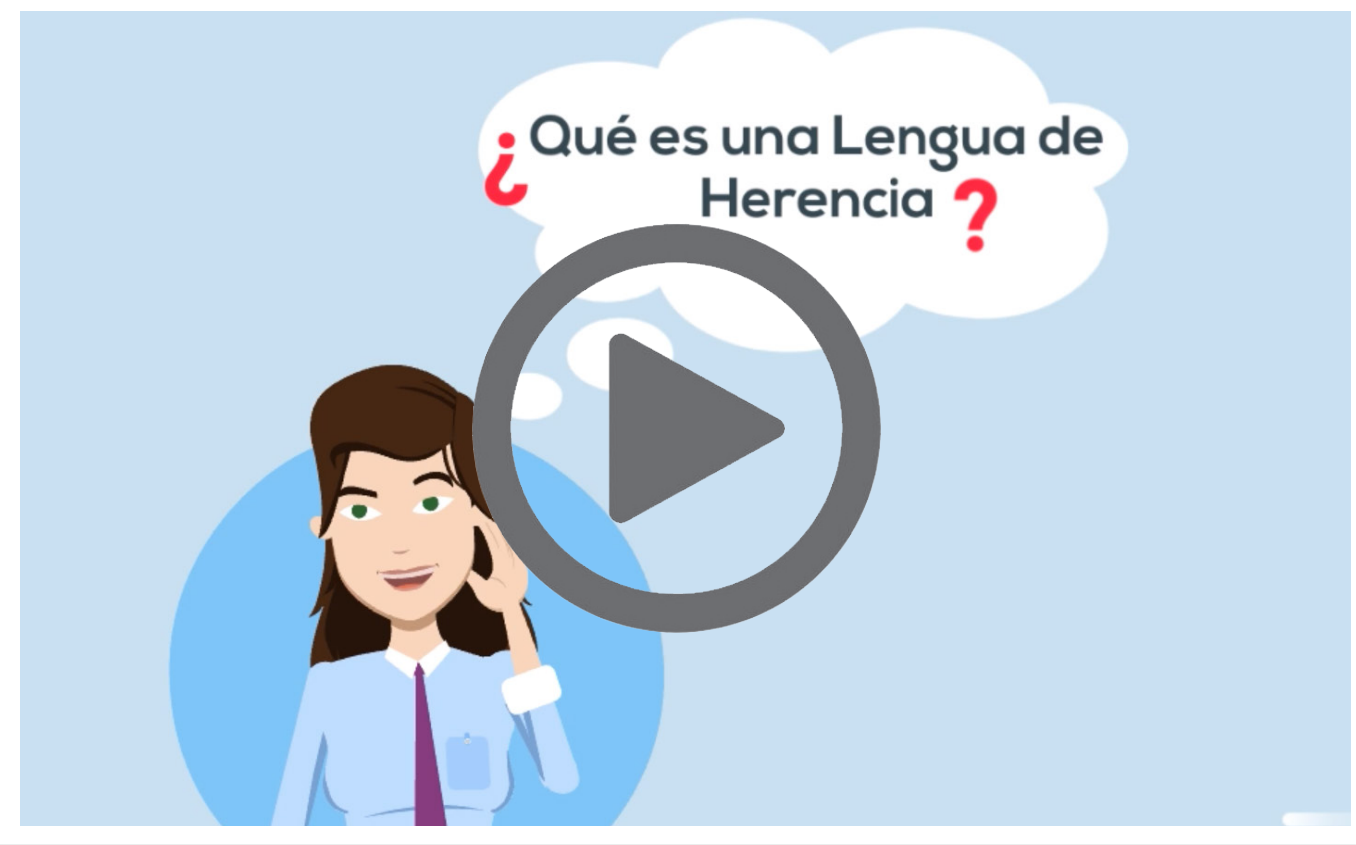


La literatura se ha referido a los hablantes de herencia como semihablantes (Wei, 2003; Gass y Selinker, 1994), término con acepciones negativas, que implica competencias comunicativas de producción incompletas en hablantes bilingües. De igual forma, se ha usado el término hablantes pasivos (Gass y Selinker, 1994; Wei 2003), que destaca, también en términos negativos, la ausencia de producción o producción reducida en una de las lenguas. Competencia comunicativa se define como "el conocimiento de las reglas de la lengua y de sus normatividades lingüísticas y extralingüísticas: el hablante, todo hablante, conoce o no conoce tales reglas, y a partir de ese conocimiento, actúa, interpreta y se interrelaciona" (Valiñas, 1986, p.47). El conocimiento de la lengua refiere a la competencia lingüística que tiene que ver con los conocimientos y habilidades gramaticales de los hablantes; en cambio, el conocimiento de las normativas lingüísticas y extralingüísticas, indica el conocimiento y habilidades en el uso de la lengua (Montrul, 2016). Todo esto es evidente cuando aprendemos una segunda lengua, proceso en el que corroboramos que saber las reglas gramaticales, así como palabras y frases en la lengua meta no significa que sepamos usar la lengua de manera adecuada, por lo que debemos aprender las reglas de uso.

Los hablantes de herencia son un tipo de hablantes bilingües que, a diferencia de los hablantes monolingües - que únicamente hablan una lengua - y de los bilingües — que hablan fluidamente sus dos lenguas-, desarrollan más habilidades en la lengua dominante de la sociedad en la que viven que en su lengua de casa o familiar. Benmamoun et al. (2013) definen a los hablantes de herencia como "hablantes bilingües, simultáneos o secuenciales, cuya L1 (lengua de casa) es severamente restringida a estímulos insuficientes [...] pueden entender la lengua de casa y hablarla hasta cierto grado pero se les facilita más la lengua dominante de su sociedad" (p. 133). Por otro lado, los hablantes bilingües simultáneos adquirieron a temprana edad y al mismo tiempo sus dos lenguas, y los hablantes bilingües secuenciales primero aprendieron una lengua y después la otra. Es importante resaltar que la definición de hablantes de herencia implica un contexto de subordinación lingüística —como en el caso de Rosa y Fernando-. Dicho contexto permite dimensionar la restricción de estímulos o estímulos insuficientes, ya que como en el caso de Fernando los mismos parientes fueron quienes decidieron dejar de hablarle en su lengua de casa, el paipai, cuando empezó a ir a la escuela.

Montrul (2010), especialista en el estudio de Hн identifica algunas de las características principales que distinguen a este tipo de bilingües:

- Habilidades débiles en su lengua de herencia, que van desde únicamente receptivas, es decir, que entienden todo o partes de lo que les dicen, hasta habilidades de producción oral de nivel intermedio a casos de nivel avanzado.

- Las gramáticas en lenguas de herencia presentan procesos de simplificación en la producción de la lengua; es decir, las reglas, marcas y valores que usa el sistema lingüístico se ven reducidos. Al parecer, el 
nivel más afectado es la flexión morfológica, la cual tienen que ver con las categorías gramaticales, como son las marcas de persona, número, tiempo, entre otras. Un ejemplo es la reducción de marcas de caso en lenguas que tienen sistema complejos de casos. Por ejemplo, en el ruso se distinguen seis marcas — nominativo (función de sujeto), acusativo (función de objeto directo), dativo (función de objeto indirecto), instrumental (función de instrumento), oblicuo (función de no sujeto)y genitivo (función de complemento)—, de las cuales los hablantes de herencia de ruso sólo usan el caso nominativo y acusativo (Montrul, 2016).

- La pronunciación de los HH es parecida a los hablantes nativos, pero también presenten características no nativas.

- El vocabulario depende de sus experiencias. Se les complica recuperar vocabulario de uso no frecuente.

Los $\mathrm{HH}$ se consideran hablantes bilingües que presentan, al mismo tiempo, características de hablantes monolingües y bilingües (Montrul, 2010). Como hablantes monolingües, los rasgos que se distinguen son la temprana exposición a la lengua, abundancia de estímulos agrupados de manera natural y el control de rasgos de la lengua adquirida a temprana edad (fonología, algo de vocabulario y algunas estructuras lingüísticas). Como hablantes bilingües, presentan transferencias de la lengua dominante a la de herencia. Por ejemplo, en hablantes de herencia de español, debido a la influencia del inglés muestran un aumento en el uso de pronombres independientes como yo, tú, él/ella, nosotros (Montrul, 2016). También presentan habilidades variables, como se explica en el siguiente apartado, y fosilización recurrente como la sobregeneralización de reglas. Un ejemplo muy común de fosilización es cuando estamos aprendiendo inglés como segunda lengua y usamos la marca $\{-e d\}$ al final de un verbo para formar el pasado, la cual no aplica para verbos irregulares.

\section{Variación en los hablantes de herencia}

Una de las características que distinguen a los Hн es la variación que presentan en sus competencias comunicativas, debido a las condiciones de adquisición y socialización a las que estuvieron expuestos, así como al dominio y a las actitudes hacia su lengua de herencia. Si las posturas hacia las lenguas de herencia por parte de los hablantes y de la comunidad son en detrimento de ésta y a favor de la lengua dominante, el uso y la exposición a la lengua de herencia se reduce, modificando el desarrollo de habilidades comunicativas en los hablantes que la adquieren.

De esta manera, la lengua de herencia suele ser exclusivamente familiar, su uso principal es en la casa o con personas del círculo social más cercano. Así ese espacio se puede considerar como un tipo de norma social, que se considera norma familiar (Ibáñez, 2020), en el que existen reglas de interacción particulares que no corresponden a las reglas de interacción fuera de ese ámbito, lo que genera variación en las habilidades comunicativas que presenta cada $\mathrm{HH}$. Es decir, al ser el espacio familiar el de mayor uso de la lengua de 
herencia, la norma de uso que se adquiere y que desarrolla es la que responde a necesidades y características de ese ámbito, lo que genera que cada uno de los HH use de manera particular su lengua de herencia. Además, el espacio familiar también es el último espacio de uso de una lengua que se encuentran en alto grado de desuso, por lo que es común que, en lenguas en peligro de extinción, existan Hн en la familia con quienes interactúen los últimos hablantes fluidos.

A causa de esta variación que presentan los Hн, se distinguen dos grandes grupos: por un lado, los HH que presentan producción, es decir aquellos que pueden hablar en su lengua de herencia; y, por otro lado, los Hн que son receptivos, los cuales se distinguen por presentar habilidades comunicativas en su lengua de familia, aun cuando tienen una producción reducida o nula. La distinción entre los dos tipos de нH se debe a que las habilidades de comprensión no implican las habilidades de producción.

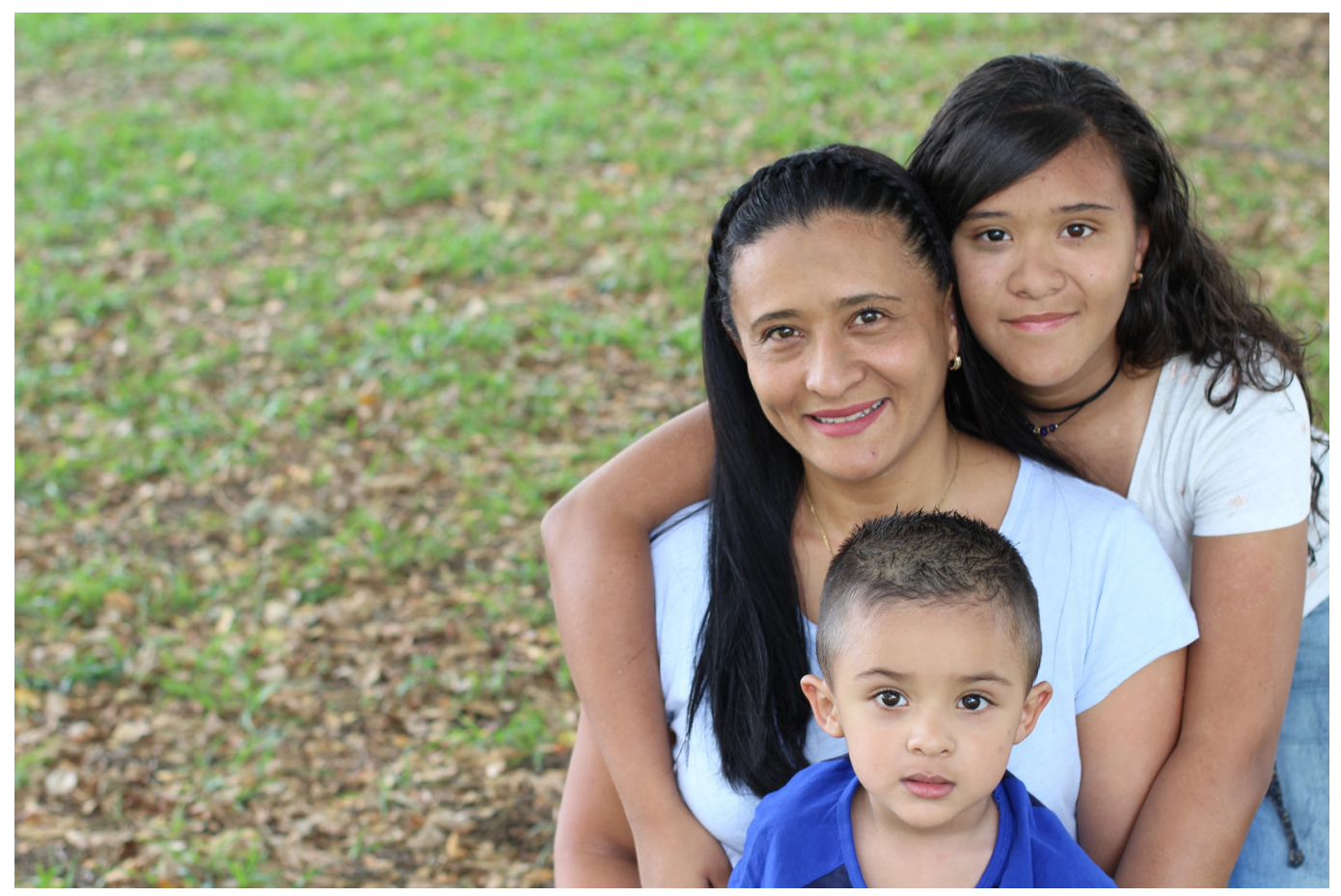

\section{Enseñanza de la lengua de herencia}

En la literatura sobre hablantes de herencia y enseñanza de segundas lenguas, el término lengua de herencia tiene dos significados. Por un lado, se usa con un sentido puramente identitario, en el que la lengua de un pueblo se concibe como un vínculo histórico, una conexión cultural con sus ancestros; en este sentido, también se conocen como lenguas ancestrales o lenguas comunitarias. Por otro, el uso del término también se refiere a la lengua que se usa con la familia, pero que no se considera como una lengua de prestigio en el lugar donde habitan los que la hablan. En contextos educativos se usa el término aprendices de lenguas de herencia para el primer significado y hablantes de herencia para el segundo. Un término más general 
es el de educación en lenguas de herencia, el cual refiere a cualquier educación en una lengua que no sea la oficial o nacional (Leeman y King, 2015).

La enseñanza de lenguas de herencia en espacios educativos formales surge desde una perspectiva de pedagogía crítica en la que se hace consciente la subordinación lingüística como condición social que subyace a este tipo de bilingüismo. Cushing (2020) menciona que los programas de enseñanza de lenguas y cultura de herencia han surgido como parte de los discursos políticos en contra de la cultura hegemónica, que amenaza la reproducción cultural e ideológica de un pueblo. La enseñanza de lenguas de herencia se convierte pues en una herramienta de resistencia imaginaria en contra de las relaciones jerárquicas de dominio, como lo indica el mismo autor.

Desde esta perspectiva, los programas de enseñanza promueven el reconocimiento de la variación lingüística. Ésta es el principal eje para la enseñanza de lenguas de herencia, desde donde se reconocen y se valoran las competencias comunicativas que presentan los HH, como es su conocimiento cultural, que forma parte de la identidad multilingüe de los estudiantes (Leeman et al., 2011).

Los programas de enseñanza de lenguas de herencia reconocen que los HH no presentan registros de habla de prestigio, ni grados altos de literacidad en su lengua de herencia. Por lo tanto, en los programas de enseñanza no se imparte la norma estándar, como sí se hace en los contextos de enseñanza de segundas lenguas (Leeman et al., 2011). Un aspecto fundamental de los programas de enseñanza de $\mathrm{HH}$ es la de lograr que los Hн reconozcan las competencias que ya poseen para valorar el conocimiento que tienen sobre su lengua de herencia (Leeman y King, 2015). Dicha postura es opuesta a los programas creados desde una ideología monolingüe dominante, que niegan la variación lingüística como estrategia, para borrar la identidad y los conocimientos lingüísticos de los hablantes (Leeman et al., 2011). De manera específica, la enseñanza de lenguas de herencia tiene como propósito ampliar las habilidades comunicativas que ya presentan los $\mathrm{HH}$, considerando el desarrollo en su precisión gramatical, sus competencias de literacidad y diversificación de los registros de habla que usan (Bernstein et al., 2010).

Un gran esfuerzo en el estudio de hablantes de herencia y en el desarrollo de programas de enseña de lenguas de herencia es el que realiza el The National Heritage Language Resource Center (El Centro de Recursos Lingüísticos para Lenguas de Herencia), con sede en la Universidad de los Ángeles, California (UCLA). En él, colaboran especialistas en diversas lenguas de herencia a nivel mundial, principalmente en contextos de migración. Asimismo, diversos esfuerzos se están dando actualmente en la enseñanza de lenguas de herencia, tanto en escuelas como en universidades de los Estados Unidos de Norteamérica. Tal es el caso de María Carreiro de la Universidad Estatal de California y Kim Potowsky (ver video 2) en la Universidad de Illinois en Chicago, quienes han desarrollado programas para la enseñanza del español como lengua de herencia, entre muchos otros investigadores. Desgraciadamente, en México no se ha impulsado el estudio de hablantes de herencia, aun cuando existe presencia de este tipo de 
Video 2. En esta conferencia, Kim Potowski habla acerca de los hablantes de herencia del español en Estados Unidos, el contexto en el que viven y el futuro del español en ese país (Instituto Cervantes, 2019). bilingüismo en la población hablante de lenguas originarias, que se encuentran en desplazamiento lingüístico.

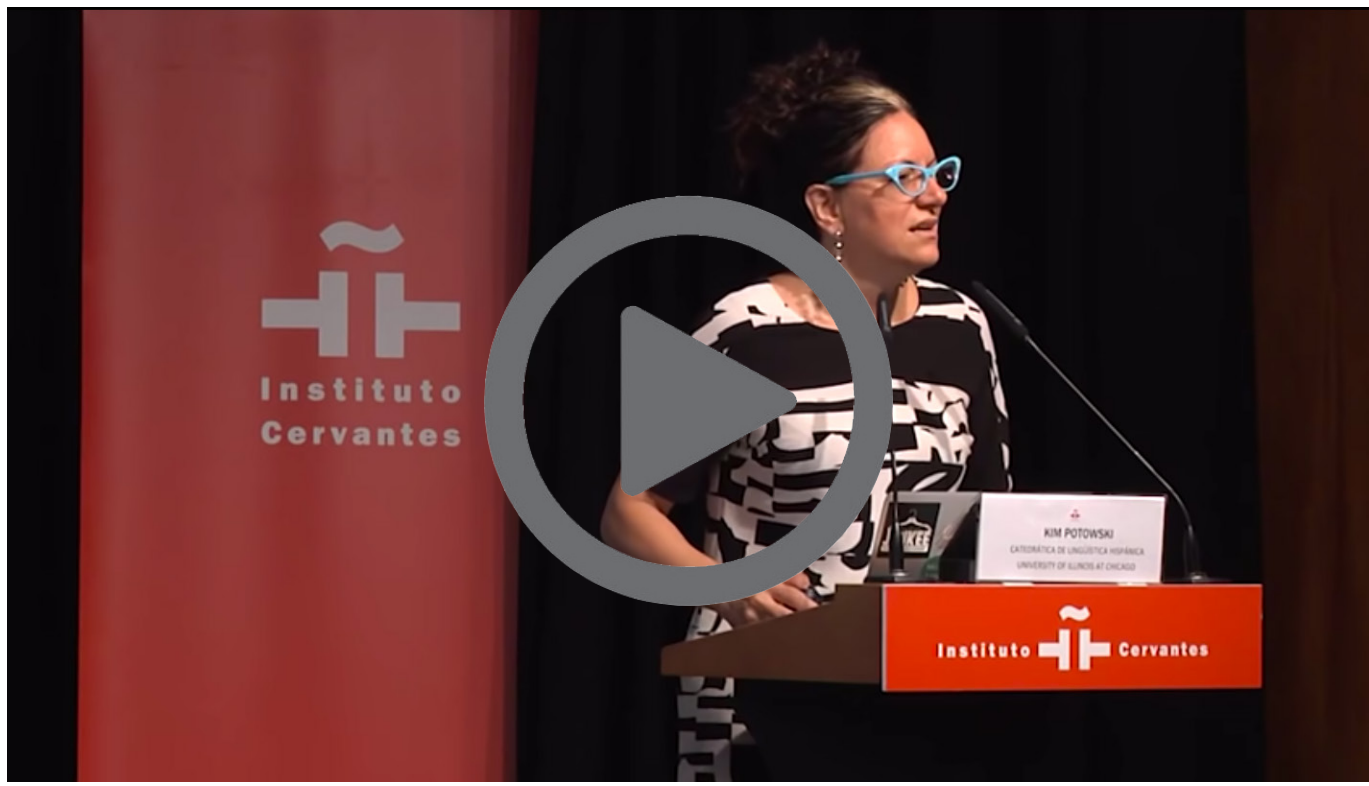

Una estrategia de enseñanza de lengua de herencia que se ha implementado consiste en realizar traducciones colaborativas (Cushing, 2020). De esta manera, se puede vincular a diversos participantes sociales con la comunidad de habla de los HH al traducir diferentes tipos de contenidos y formatos para su comunidad. Además de que evidencia el conocimiento que tienen los Hн de su lengua y de su cultura.

Para hablantes de lenguas de herencia cuya lengua ha sido poco estudiada y de la cual se cuenta con poco material escrito, como es la situación en la que se encuentran la mayoría de las lenguas originarias en México, la estrategia de inmersión conocida como método maestro-aprendiz, desarrollado por Hinton (2002), se considera como una opción viable para la enseñanza de lenguas de herencia. El método maestro-aprendiz consiste en hacer parejas, de un hablante de la lengua de interés y alguien que busque estudiarla. El aprendiz pasa tiempo con el hablante de manera cotidiana y realizan actividades únicamente usando la lengua meta. La dinámica de inmersión es similar a las condiciones en las que adquirimos nuestra lengua materna (L1), por lo que este método se percibe ideal una vez que el $\mathrm{HH}$ esté dispuesto a aprender su lengua de herencia y que el hablante fluido esté dispuesto a enseñarla.

\section{Reflexiones}

A manera de conclusión, en México el Instituto Nacional de lenguas Indígenas (INALI) reconoce oficialmente 364 lenguas-variantes nacionales que se hablan en México además del español (INALI, 2009). De éstas, 170 lenguas-variantes se encuentran en muy alto y alto riesgo de desaparición (INALI, 2012), o sea, casi la 
Video 3. Las lenguas índigenas en México (TV UnAm, 2019). mitad de la diversidad lingüística del país. Dicha situación requiere de cambios urgentes en las políticas que permitan asegurar los derechos lingüísticos de todos los hablantes de las lenguas nacionales.

El reconocimiento y visibilización de los Hн permite resaltar las condiciones de subordinación en que se encuentran los hablantes de lenguas indígenas y cuestionar las políticas lingüísticas que han generado dichas condiciones (ver video 3). Además, al identificar a los hablantes de herencias como un tipo de bilingüismo, es posible ampliar las estrategias de atención de lenguas que se encuentran en grave peligro de extinción, pues por las características que presentan los HH se puede diversificar las estrategias para promover el uso de las lenguas que se han dejado de hablar. Incluso los HH son un bilingüismo que está modificando las estrategias de enseñanza de lenguas, al valorar el papel de la lengua como parte de la identidad e ideología de la comunidad de habla en los programas de enseñanza de lenguas.

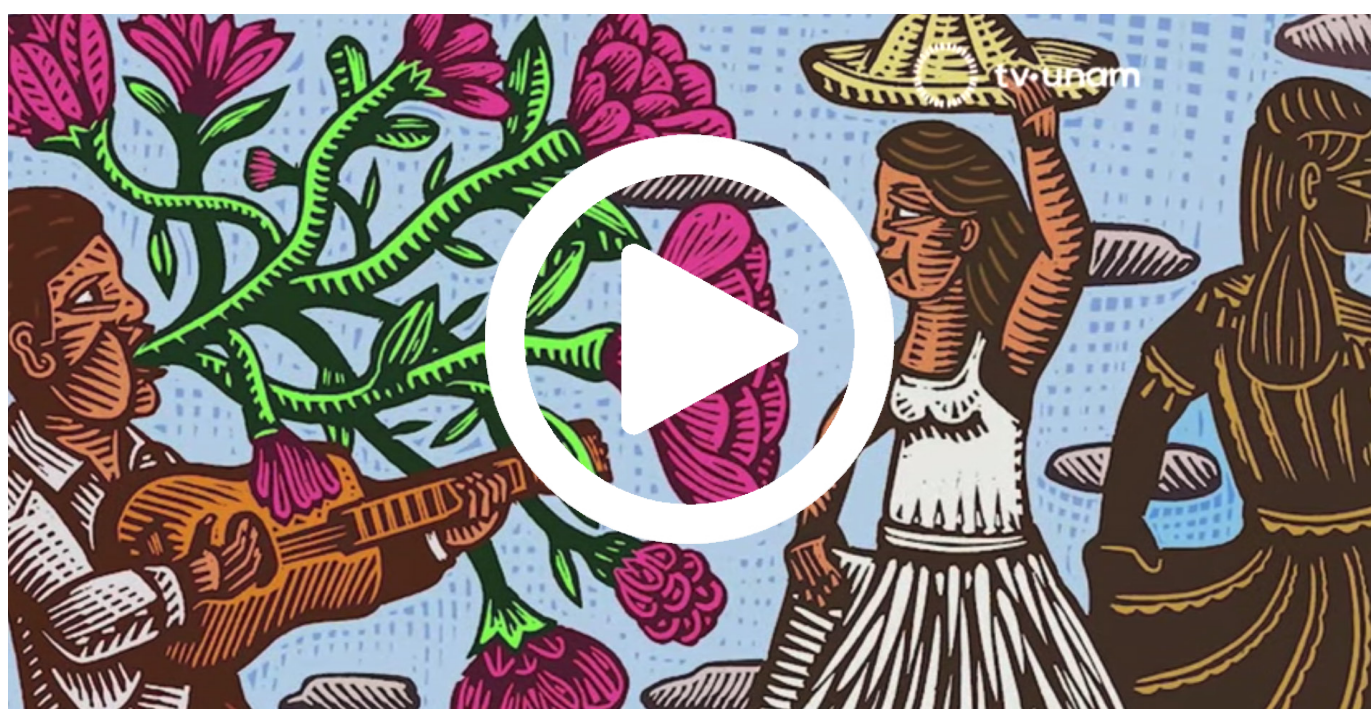

Así pues, visibilizar a los hablantes de herencia en los contextos multilingües e incluirlos en el diseño e implementación de políticas lingüísticas puede permitir que Rosa y Fernando no sean los últimos hablantes bilingües en su familia o en su comunidad de habla, sino los primeros en reivindicar su lengua.

\section{Referencias}

* Bernstein S., Burke, K., Favre, L. y Delcourt, J. (2010). Recognizing the needs and talents of the heritage language learner. Educator's Voice: NYsut's Journal of Best Practices in Education, 3, 66- 73. https://cutt.ly/9nYjYhm

* Cursos Español Colorado. (2019, 13 de abril). Hablantes de Herencia [video]. YouTube. https://youtu.be/ElnDwZ66b6k 
- Cushing, J. (2020). Heritage language education. A global view. En S. Laviosa y M. González-Davies (Eds.), The Rougtledge Handbook of Translation and Education (pp. 303-322). Routledge.

* Gass, S. M. y Selinker, L. (2008). Second language acquisition: An introductory course (3. ${ }^{a}$ ed). Routledge; Taylor and Francis Group.

* Hinton, L. (2002). How to keep your language alive a commonsense approach to oneon-one language learning. Heyday Books.

* Ibáñez, E. (2020). Diagnóstico de competencias comunicativas de hablantes de herencia en la lengua paipai [Tesis de Maestría en Lenguas Modernas, Universidad Autónoma de Baja California, México].

* Instituto Cervantes. (2019, 1 de agosto). El futuro de la lengua española en Estados Unidos [video]. YouTube. https://youtu.be/Jdp_KEDIC4Y

* Instituto Nacional de Lenguas Indígenas (INALI). (2009). Catálogo de las Lenguas Indígenas Nacionales. INALI.

* Instituto Nacional de Lenguas Indígenas (InalI). (2010). Proyecto inicial de Documentación de las lenguas de la familia lingüística cochimí-yumana [informes]. INALI.

* Leeman, J., Rabin, L. y Roman-Mendoza, E. (2011). Identity and Activism in Heritage Language Education. The Modern Language Journal, 95(4), 481-495. https://doi. org/10.1111/j.1540-4781.2011.01237.x

* Leeman, J. y King, K. (2015). Heritage Language Education: Minority Language Speakers, Second Language Instruction, and Monolingual Schooling. En M. Bigelow y J. Ennser-Kananen (Eds.). The Routledge Handbook of Educational Linguistics (pp. 210-223). Routledge.

- Montrul, S. (2010). Current Issues in Heritage Language Acquisition. Annual Review of Applied Linguistics, 30, 3-23. https://doi.org/10.1017/S0267190510000103

* Montrul, S. (2016). The acquisition of heritage languages. Cambridge University Press.

* Polinsky, M. (2018). Heritage Languages and their Speakers. Cambridge University Press.

* tV unam. (2019, 30 de agosto). RESCATE DE LENGUAS INDÍGENAS [video]. YouTube. https://youtu.be/Yf1X8sycxnM

* United Nations Educational, Scientific and Cultural Organization (UnEsco). (2003). Vitalidad y peligro de desaparición de las lenguas. Reunión Internacional de Expertos sobre el programa de la unESCO Salvaguardia de las Lenguas en Peligro. unESCO.

* Valiñas, L. (1986). La alfabetización y su problemática: El caso mixe [Tesis de Maestría en lingüística, Escuela Nacional de Antropología y Historia].

* Wei, L. (Ed). (2000). The Bilingualism Reader. Routledge. 


\section{Cómo CITAR ESTE ARTículo}

* Ibáñez Bravo, María Elena. (2021, julio-agosto). Los últimos serán los primeros: la importancia de los hablantes de herencia. Revista Digital Universitaria (RDU), 22(4). http://doi.org/10.22201/cuaieed.16076079e.2021.22.4.6 